\title{
Evaluation of a Novel Endobronchial Ultrasound-Guided Lymph Node Forceps in Enlarged Mediastinal Lymph Nodes
}

\author{
Kaid Darwiche $^{c}$ Lutz Freitag $^{c}$ Arun Nair $^{\mathrm{a}}$ Christiane Neumann ${ }^{\mathrm{b}}$ \\ Ruediger Karpf-Wissel ${ }^{c}$ Stefan Welter ${ }^{d}$ Jeremias Wohlschlaeger ${ }^{e}$ \\ Dirk Theegarten ${ }^{\mathrm{e}}$
}

${ }^{a}$ Department of Respiratory Medicine, Freeman Hospital, Newcastle upon Tyne, UK; ${ }^{b}$ Department of Internal Medicine, Kreisklinik Bad Neustadt, Bad Neustadt, and Departments of ' Interventional Pneumology and

${ }^{\mathrm{d}}$ Thoracic Surgery, Ruhrlandklinik, and 'Institute of Pathology and Neuropathology, University Hospital of Essen, University of Duisburg-Essen, Essen, Germany

\section{Key Words}

Bronchoscopy · Endobronchial ultrasound · Lung cancer · Mediastinal lymph node · Sarcoidosis · Ultrasound-guided biopsy. Transbronchial needle aspiration

\section{Abstract \\ Background: Endobronchial ultrasound-transbronchial nee- dle aspiration (EBUS-TBNA) is a useful technique for cytolog- ical assessment of enlarged mediastinal lymph nodes with a high diagnostic yield for lung cancer. However, the small sample volume can be problematic in diagnosing benign diseases and for molecular analysis of malignant tumours. Objectives: The aim of the study was to evaluate a novel lymph node forceps for EBUS-guided lymph node biopsy (EBUS-transbronchial forceps biopsy; EBUS-TBFB) in malig- nant and benign conditions concerning safety, feasibility, and diagnostic yield. Methods: Patients with enlarged medi- astinal or hilar lymph nodes were included. EBUS-TBNA was performed followed by EBUS-guided TBFB with the lymph node forceps. Three biopsy specimens were obtained. The diagnostic yields of EBUS-TBFB, EBUS-TBNA, and the combi- nation of both sampling techniques were compared. Com-}

plications were systematically recorded. Results: Fifty-five patients with enlarged mediastinal nodes were enrolled into this study. Specimens adequate for histological analysis were obtained in all but one case using EBUS-TBFB. EBUSTBFB increased the diagnostic yield of EBUS-TBNA from 64 to $93 \%$ in benign conditions. The overall diagnostic yield was higher compared to EBUS-TBNA alone. EGFR mutation analysis could be achieved in the forceps biopsy samples as needed. No complications were observed. Conclusions: EBUS-TBFB with a novel lymph node forceps is safe and provides adequate histological specimens of enlarged mediastinal lymph nodes. EBUS-TBFB increases the diagnostic yield in benign conditions and may add value in molecular analysis of non-small cell lung cancer.

Copyright @ 2013 S. Karger AG, Basel

\section{Introduction}

Endobronchial ultrasound-transbronchial needle aspiration (EBUS-TBNA) has been established as a routine method for mediastinal and hilar lymph node evaluation. The diagnostic yield is high, particularly in proving and

\section{KARGER}

E-Mail karger@karger.com

www.karger.com/res
(C) 2013 S. Karger AG, Basel

0025-7931/13/0863-0229\$38.00/0
Dr. med. Kaid Darwiche

Department of Interventional Pneumology Ruhrlandklinik, University of Duisburg-Essen Tüschener Weg 40, DE-45239 Essen (Germany)

E-Mail kaid.darwiche@ ruhrlandklinik.uk-essen.de 
staging of non-small cell lung cancer (NSCLC) [1-4]. However, a shortcoming of this method is its low negative predictive value reported in some studies. Guidelines recommend confirmation of negative results by surgical procedures [5].

The diagnostic value of EBUS-TBNA in the evaluation of mediastinal lymphadenopathy in conditions other than lung cancer is less clear. Its accuracy in establishing the correct histopathological diagnosis of lymphoma is lower than for lung cancer. Mediastinoscopy is frequently required in order to obtain sufficient tissue for critical treatment decisions $[6,7]$. For sarcoidosis, some authors consider the diagnostic yield of EBUS-TBNA good enough while others report inferior results and recommend more invasive tissue sampling procedures $[3,8,9]$. EBUS-TBNA, therefore, has some limitations in the assessment of mediastinal lymphadenopathy. One plausible reason might be the small sample volume of tissue obtained by EBUS-TBNA performed with the 22-gauge needle. A histologic biopsy taken with a forceps may therefore have additional value.

Some investigators have previously reported the use of biopsy forceps through the hole made by the TBNA needle for obtaining histologic material from enlarged subcarinal lymph nodes either blindly or under fluoroscopic or EBUS guidance [10-13]. Recently, in a study in patients with unexplained mediastinal lymphadenopathy, an ultrasoundguided forceps yielded improved diagnostic results [14]. However, the described methods require considerable technical skill and bronchoscopic expertise. Furthermore, mainly subcarinal lymph nodes were assessed. In another study, a needle forceps was used without EBUS guidance to sample subcarinal lymph nodes without first puncturing the tracheal wall with a standard TBNA needle. However, in this study, in $28 \%$ of cases, it was impossible to penetrate the wall with the needle forceps [15].

The aim of the present study was to develop an easyto-use dedicated two-in-one lymph node forceps for sampling from all EBUS scope accessible mediastinal or hilar lymph nodes. The utility, safety, and diagnostic yield of this needle forceps were prospectively tested in a clinical trial.

\section{Material and Methods}

\section{Lymph Node Forceps}

The challenge was to get an instrument that is small and sharp enough to penetrate the tracheobronchial wall but big enough to obtain a large volume specimen. The handpiece and the wires must be strong enough that the forceps can be opened and closed inside

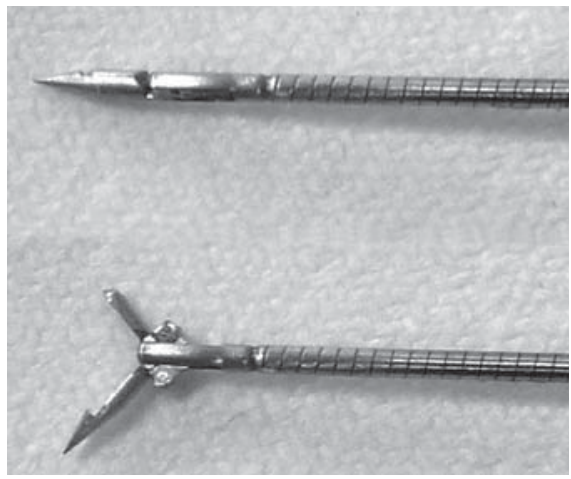

Fig. 1. The newly developed lymph node forceps is shaped as a needle in a closed position to penetrate the bronchial mucosa.

a lymph node without getting too big for the working channel of the bronchoscope. We tested various prototypes in excised pig lungs until eventually a company $\left(\mathrm{MTW}^{\circledR}\right.$ Endoskopie, Wesel, Germany) provided us with a CE-marked model, suitable for clinical testing (fig. 1). This specifically designed lymph node forceps is shaped like a needle in the closed position with a sharpened bevelled tip and housed in an outer sheath to prevent damage to the working channel of the bronchoscope. It is not possible to fix the instrument at the bronchoscope. Therefore, the bronchoscopist has to take care not to push the needle forceps forward before the sheath is visible outside the working channel. Once pushed out of the sheath, the needle forceps is sharp enough to omit previous perforation of the bronchial wall with a 19-gauge needle.

\section{Patients}

Patients were eligible for inclusion if they were 18 years or older and had enlarged (>10 mm) mediastinal or hilar lymph nodes on computed tomography. Consecutive patients referred for EBUS-TBNA between February and May 2011 and between March and July 2012 were enrolled into this prospective single-centre study if a diagnosis of lung cancer, sarcoidosis, or lymphoma was suspected based on clinical and radiographic findings. Negative or ambiguous endoscopic histological results were confirmed by surgical procedures (mediastinoscopy, open thoracotomy) and clinical follow-up (including computed tomography) over 6 months. The study was approved by the Ethics Committee of the University of Duisburg-Essen (approval No. 10-4520). Written informed consent was obtained from all patients. The study was registered at the German Cancer Trials Registry (No. 591).

Endobronchial Ultrasound-Transbronchial Needle Aspiration

After conventional flexible bronchoscopy, EBUS-TBNA examination was performed under general anaesthesia as described previously with an EBUS-TBNA bronchoscope (model BF-UC 180F; Olympus, Japan), which was connected to an ultrasound scanner (EU-ME1; Olympus) [3]. All mediastinal and hilar lymph node stations were assessed systematically and punctured 3 times with a 22-gauge needle (NA-2015X-4022; Olympus) if their diameter exceeded $5 \mathrm{~mm}$. EBUS-TBNA was performed by interventional bronchoscopists (K.D., L.F., and R.K.-W.) who are experienced in this field. 


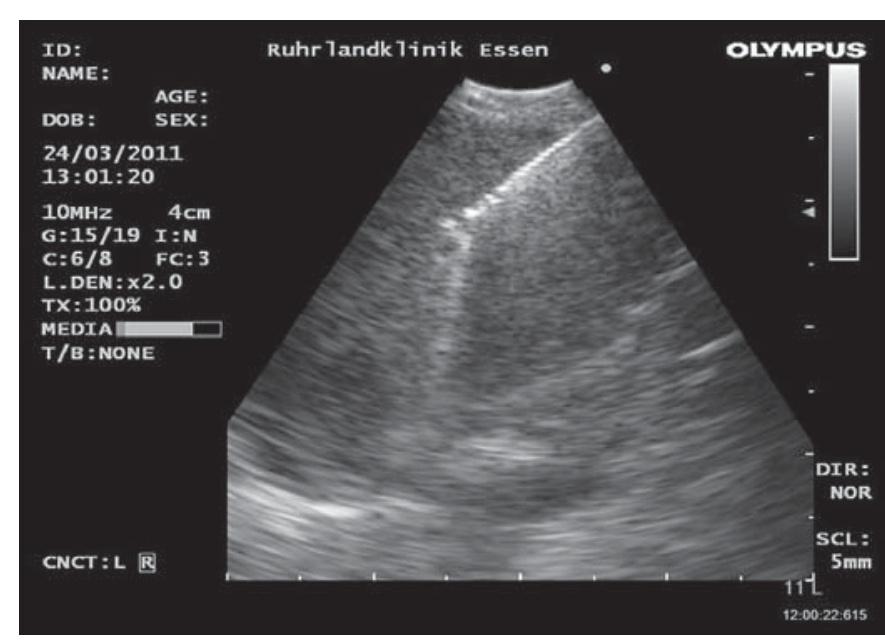

Fig. 2. Positioning, opening, and closure of the cups of the lymph node forceps can easily be supervised on the ultrasound image.

\section{EBUS-Transbronchial Forceps Biopsy}

After EBUS-TBNA, a single enlarged lymph node was identified as the index lymph node for transbronchial forceps biopsy (TBFB) by the endoscopist. TBFB was obtained only from the index lymph node, because performing TBFB in all enlarged lymph nodes would have extended the examination time too much. The size of this index lymph node was divided into 3 groups according to its short axis diameter $(10-20 \mathrm{~mm}, 21-30 \mathrm{~mm}$, and $31 \mathrm{~mm}$ and greater). When appropriate, colour Doppler ultrasound mode was used to exclude vessels in the target area. The jabbing method of TBNA was used for penetrating the bronchial wall and puncturing the target lymph node [16]. With the forceps close to the orifice, the working channel of the bronchoscope helped to stabilise the device and facilitated tissue penetration. Once inside the lymph node, the forceps was opened and moved slightly back and forth to achieve complete opening against tissue resistance. Finally, the lymph node forceps was advanced slightly, closed, withdrawn, and retracted into the outer sheath. All of the above steps were performed under ultrasound guidance (fig. 2). Three specimens were obtained. Patients were observed for $2 \mathrm{~h}$ after the procedure and a chest X-ray was performed to exclude pneumothorax or other procedure-related adverse effects.

\section{Pathology}

The aspirated material of the EBUS-TBNA was smeared onto glass slides and fixed with $95 \%$ alcohol. Additional aspirated material was placed into a tube containing CytoLyt ${ }^{\circledR}$ solution (Hologic Inc., USA). Rapid on-site pathologic evaluation (ROSE) was not performed in this study. The tissue specimens obtained by the lymph node forceps were fixed in 3.5\% buffered formaldehyde solution. After hematoxylin and eosin (H\&E) staining, cytology and histology were evaluated independently by two experienced pathologists. A positive cytological or histological result of malignancy was accepted as evidence of cancer. Immunohistochemistry and assessment of EGFR mutation were performed if needed. De-

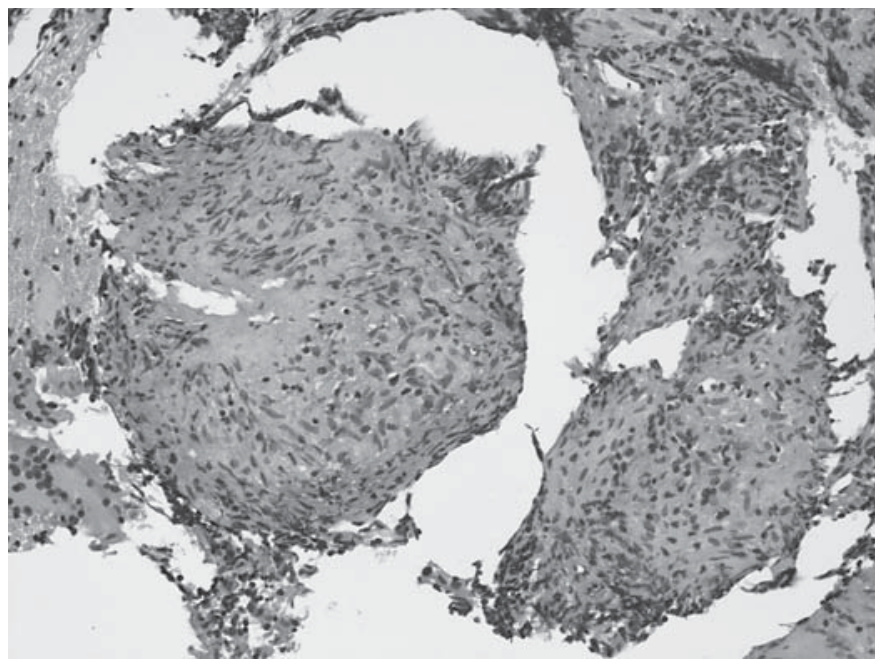

Fig. 3. EBUS-TBFB of a mediastinal lymph node. Histology reveals aggregates of epithelioid cells, typical for sarcoidosis. H\&E. Original magnification $\times 200$.

tections of groups of epithelioid cells in cytological probes or noncaseating epithelioid granulomas in histological specimens were regarded as proof of sarcoidosis when other granulomatous diseases could be excluded and clinical and radiological findings supported this diagnosis (fig. 3). The finding of epithelioid caseating granulomas was considered diagnostic for tuberculosis if clinical and radiological findings supported this diagnosis and polymerase chain reaction was positive for Mycobacterium tuberculosis complex [17].

\section{Statistical Analysis}

The sensitivity, specificity, and predictive values were calculated using the standard definitions. The diagnostic yields of EBUS-TBNA and a combined approach with EBUS-TBNA and EBUS-TBFB were compared with the McNemar test for dependent samples. $\mathrm{p}<0.05$ was considered statistically significant. Statistics analysis was performed using SPSS version 19.0 (SPSS Inc., Chicago, Ill., USA).

\section{Results}

The study population consisted of 55 patients who met the inclusion criteria. Seven patients were excluded from the analysis as they withdrew consent to invasive procedures or follow-up (fig. 4). The mean age of the 48 patients enrolled per protocol was 54 years (range 19-79). There were 26 males and 22 females within the cohort. The respective index lymph nodes chosen for EBUSTBFB by the endoscopist were located most often in the subcarinal (station 7) or right hilar (station 11R) region. In the majority of cases, the size of the index lymph node 


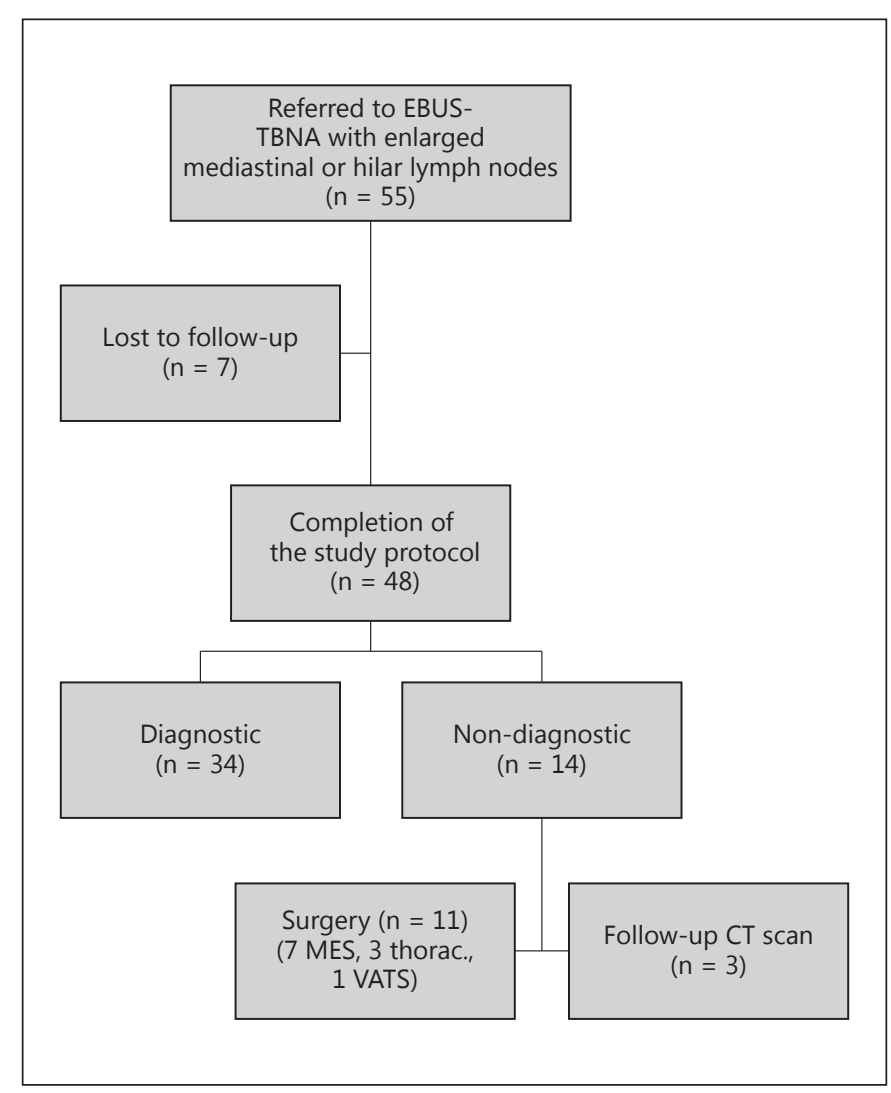

Fig. 4. Flow chart showing enrolment and analysis of 55 patients who were registered in the study, 48 of whom were eligible for analysis. VAT $=$ Video-assisted thoracic surgery.

did not exceed $20 \mathrm{~mm}$ (table 1). The bronchial wall was successfully penetrated by the lymph node forceps in all cases. Three tissue specimens were obtained from all but one lymph node, in which only 2 samples were taken. Adequate histologic specimens were obtained from all patients except the first patient recruited into the study. EBUS-TBNA revealed features compatible with a diagnosis of sarcoidosis in this patient.

In 32 patients (67\%), a classifying diagnosis was made with EBUS-TBFB alone (table 1). The pathologic analysis revealed NSCLC in 12 cases. Small cell lung cancer (SCLC) and a relapse of oesophageal cancer were diagnosed in 2 and 1 patient, respectively. Sixteen patients had a granulomatous inflammatory reaction and, considering the clinical phenotype, sarcoidosis was the final diagnosis. In 1 patient, caseating granulomas and a positive polymerase chain reaction for $M$. tuberculosis complex confirmed tuberculosis.
Table 1. Distribution, size, and pathologic results of mediastinal and hilar lymph nodes examined by EBUS-TBFB

\begin{tabular}{lr}
\hline Lymph node station & \\
$2 \mathrm{R}$ & 2 \\
$4 \mathrm{R}$ & 7 \\
$4 \mathrm{~L}$ & 4 \\
7 & 15 \\
$10 \mathrm{~L}$ & 3 \\
$11 \mathrm{R}$ & 10 \\
$11 \mathrm{~L}$ & 7 \\
Size & 22 \\
$\geq 10-20$ mm & 13 \\
$\geq 21-30$ mm & 13 \\
$\geq 31$ mm & 12 \\
Pathologic results & 2 \\
$\quad$ NSCLC & 1 \\
SCLC & 16 \\
Oesophageal cancer & 1 \\
Sarcoidosis & 13 \\
Tuberculosis (PCR) & 2 \\
Normal lymph node tissue/questionable result & 1 \\
Anthracotic lymph node & \\
Inadequate biopsy & \\
\hline & \\
PCR = Polymerase chain reaction. & \\
\hline
\end{tabular}

In 16 patients, EBUS-TBFB did not provide a classifying diagnosis. Sarcoidosis and large cell lung carcinoma was diagnosed by the result of EBUS-TBNA in 1 patient, respectively. The remaining patients underwent further examination by surgical means (11 patients) or follow-up CT (3 patients) (fig. 4). This revealed NSCLC in 3 cases, anthracosilicosis in 2, and Castleman's disease, bronchogenic cyst, and sarcoidosis in 1 patient, respectively. In the remaining 6 patients, surgical evaluation and followup confirmed the benign condition.

\section{Diagnostic Accuracy}

The diagnostic accuracy of EBUS-TBNA for malignant and benign diseases was $80 \%(16 / 20)$ and $64 \%$ (18/28), respectively. Lung cancer (NSCLC and SCLC) was diagnosed correctly by EBUS-TBNA in 15 of 18 patients $(83 \%)$. The only patient with lymphoma (Castleman's disease) could not be detected by EBUS-TBNA. EBUS-TBNA was able to identify $61 \%$ of patients with sarcoidosis and the only patient with tuberculosis. Overall, 34 of 48 patients (71\%) were classified correctly by EBUS-TBNA.

As we performed EBUS-TBNA and EBUS-TBFB of the same index lymph node, we can reliably compare these methods. EBUS-TBFB significantly improved the 
Table 2. Diagnostic yield of EBUS-TBNA, EBUS-TBFB, and a combined approach

\begin{tabular}{lcccc}
\hline & EBUS-TBNA & EBUS-TBFB & Combined & p value $^{\mathrm{a}}$ \\
\hline Overall diagnostic accuracy & $34 / 48(71 \%)$ & $40 / 48(83 \%)$ & $42 / 48(88 \%)$ & n.s. \\
Malignant & $16 / 20(80 \%)$ & $15 / 20(75 \%)$ & $16 / 20(80 \%)$ & n.s. \\
NSCLC & $13 / 16(81 \%)$ & $12 / 16(75 \%)$ & $13 / 16(81 \%)$ & n.s. \\
SCLC & $2 / 2(100 \%)$ & $2 / 2(100 \%)$ & $2 / 2(100 \%)$ & n.s. \\
Other than NSCLC & $1 / 2(50 \%)$ & $1 / 2(50 \%)$ & $1 / 2(50 \%)$ & n.s. \\
Benign & $18 / 28(64 \%)$ & $25 / 28(89 \%)$ & $26 / 28(93 \%)$ & $<0.05$ \\
Sarcoidosis & $11 / 18(61 \%)$ & $16 / 18(89 \%)$ & $17 / 18(94 \%)$ & $<0.05$ \\
Anthracosilicosis & $0 / 2$ & $2 / 2$ & $2 / 2$ & \\
Tuberculosis & $1 / 1$ & $1 / 1$ & $1 / 1$ & \\
Bronchogenic cyst & $0 / 1$ & $0 / 1$ & $6 / 1$ & \\
Reactive lymphadenopathy & $6 / 6$ & $6 / 6$ & $6 / 6$ & \\
\hline
\end{tabular}

a EBUS-TBNA vs. a combined approach.

diagnostic yield in benign conditions compared to EBUSTBNA (table 2). Of the 18 patients with sarcoidosis, in 6 patients $(33 \%)$ the diagnosis could be made only by EBUS-TBFB. EBUS-TBNA was not diagnostic in these cases.

In patients with lung cancer, sensitivity was not improved with EBUS-TBFB compared to EBUS-TBNA. However, in 1 patient EBUS-TBFB resulted in reclassification of the diagnosis of adenocarcinoma on EBUS-TBNA to large cell carcinoma on EBUS-TBFB. Analysis of EGFR mutation was performed in 3 of the samples and revealed a wild type in all. The sensitivity, specificity, positive and negative predictive value, and accuracy for EBUS-TBNA and EBUS-TBFB were calculated as 85, 100, 100,43 , and $88 \%$, respectively.

The bronchoscopic examination time was extended by the EBUS-TBFB procedure by 6 min (range 2-15).

\section{Complications}

No clinical complications or damages of the bronchoscopes due to TBFB were observed.

\section{Discussion}

In this prospective comparative trial, we have demonstrated the safety and clinical effectiveness of a novel twoin-one lymph node forceps for EBUS-guided TBFB.

EBUS-TBNA has been extensively evaluated in diagnosing and staging of NSCLC. This diagnostic procedure has an excellent yield in normal sized and enlarged mediastinal and hilar lymph nodes, with a sensitivity of 84-
94\% [3, 4, 18-20]. In this study, the diagnostic yield of EBUS-TBNA was marginally lower than reported before in bigger studies. Technologies resulting in an increased output of cell block material could perhaps improve the overall detection rates of malignant diseases. In our study, EBUS-TBFB demonstrated equivalent results and confirmed mediastinal metastasis in 12 of 16 cases $(75 \%)$ with NSCLC.

In one case, the final diagnosis changed from adenocarcinoma to large cell carcinoma after evaluation of the specimen obtained by the lymph node forceps. In this regard, it is essential to recognise that personalised chemotherapy regimens must be based on accurate molecular subtyping of NSCLC. It can be expected that this aspect will gain importance in the future with further development of molecular markers. It has been shown that tissue samples obtained by EBUS-TBNA are sufficient for molecular testing such as EGFR mutation or gene fusions/ translocations such as EML4-ALK mutation [21, 22]. However, nobody can argue that more material volume enables pathologists to do more molecular tests and that this could result in a more specific treatment.

The diagnostic value of EBUS-TBNA, which provides cell groups for a cytological evaluation instead of tissue for histological analysis in the majority of the cases, is less well established in patients with sarcoidosis or other benign diseases $[3,6,8,9,23]$. The reason for a suboptimal diagnostic hit rate may be the small specimen size by EBUS-TBNA that does not allow adequate pathological analysis because the general tissue architecture cannot be evaluated. The use of mini-forceps guided by EBUS has been evaluated by other investigators. However, there are 


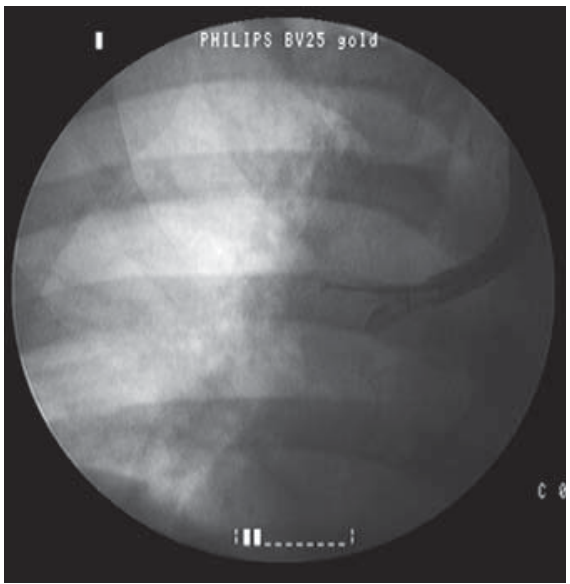

Fig. 5. The lymph node forceps passes the bronchial wall, fixed by the EBUS bronchoscope and using the opposite bronchial wall as a counterforce.

significant practical difficulties using these devices. The technique used is cumbersome, being a 2-stage process wherein a hole is first created in the bronchial wall using a 19-gauge needle and the mini-forceps is subsequently introduced into the subcarinal lymph node. The diagnostic yield with such a procedure is increased with respect to Hodgkin disease, non-Hodgkin lymphoma, and sarcoidosis [10-13]. In a recent study using another type of forceps, diagnostic sensitivity increased to $82 \%$ when EBUS-TBNA and EBUS-guided forceps biopsies were combined [14]. However, these studies have been performed mainly in subcarinal nodes, and the blunt forceps did not pass the perforated mucosa and penetrate the lymph node in all cases. The need for a second instrument (TBNA-needle and mini-forceps) and a second bronchoscope is time consuming and financially detrimental. Therefore, there is a need for a reliable two-inone instrument which can puncture the mucosa and take forceps biopsies. One such device developed by Gasparini et al. [15] achieved a diagnostic yield of $57.1 \%$. The limitation of this study was that it did not use EBUS guidance and consequently failed to obtain adequate specimens in $28.5 \%$ of cases [15]. In a more recent study, Herth et al. [24] reported the use of a two-in-one forceps device (manufactured by Olympus Medical Systems Corp., Japan) with EBUS guidance. They reported an overall diagnostic yield of $86 \%$ and were unable to obtain adequate histologic specimens in $10 \%$ of cases [24]. The applied instrument seems to be comparable to the one used in this study. However, a direct comparison between these two devices is not possible as the former is not commercially available. Using the opposite bronchial wall as a counterforce (fig. 5), our lymph node forceps could pass through the bronchial wall in all cases. Only in the first patient recruited for the study did the lymph node forceps not penetrate the lymph node after passing the mucosa, and the obtained material was considered inadequate for histological evaluation. With further experience, the lymph node forceps reached the lymphatic tissue in all other cases.

Previous studies have indicated that EBUS-TBNA can have a diagnostic yield in sarcoidosis of $54-93 \%$ at least in experienced centres $[8,9]$. A recent meta-analysis of Agarwal et al. [25] in 553 patients suggests an excellent overall diagnostic yield of EBUS-TBNA in this field. We observed a sensitivity of $61 \%$ in sarcoidosis patients. This lower value can be attributed to some possible reasons. First, we did not use rapid onsite cytology which could have improved the diagnostic yield of EBUS-TBNA. Second, only 3 biopsies have been obtained from the index lymph node. Garwood et al. [9] found that the yield exceeded $80 \%$ at 5 passes, whereas it was below $70 \%$ at 3 passes. Third, the diagnosis of sarcoidosis has to be evaluated carefully. The detection of isolated epithelioid cells cannot be regarded as adequate to diagnose sarcoidosis on its own because these findings are also seen in reactive changes. With the additional use of the lymph node forceps, an increased sensitivity of $94 \%$ could be achieved. Our results were comparable to those obtained by other investigators who used a similar device $[10,24]$. If mediastinal lymph nodes are affected by sarcoidosis, mediastinoscopy remains an option when other bronchoscopic procedures have not been diagnostic [26, 27]. However, hilar lymph nodes, which are mostly affected by this disease, are not accessible by standard cervical mediastinoscopy. In addition, mediastinoscopy is an invasive procedure and is therefore associated with a higher morbidity compared to EBUS-TBNA [28, 29]. Our findings suggest that lymph node forceps may be useful, especially in patients suspected to have a benign disease as the use of TBFB avoids a more invasive surgical diagnostic procedure in $6(13 \%)$ of the cases.

Clinicians demand diagnostic techniques that are safe and effective. This is even more relevant when evaluating new endoscopic biopsy devices, which attempt to obtain additional lymph nodal tissue and are therefore more invasive than conventional TBNA. EBUS-TBNA has low morbidity and mortality compared to cervical mediastinoscopy [27, 30, 31]. Therefore, each novel method or device has to be proven on safety issues. In our study of a 
new EBUS-guided lymph node forceps, no complications in patients or with endoscopes were observed. However, the presence of blood vessels in the target area of EBUSTBFB should be carefully investigated by Doppler sonography.

It is important to point out that EBUS-TBFB is a promising technique, but it does require advanced bronchoscopic skills and leads to an extended examination time, albeit minimal. One limitation of this study is the lack of rapid onsite evaluation of the TBNA specimens, which could have improved the diagnostic yield of EBUS-TBNA.
In conclusion, the use of a dedicated two-in-one lymph node forceps to perform EBUS-guided TBFB is feasible, safe, and it provides histologic specimens even of slightly enlarged mediastinal or hilar lymph nodes. The diagnostic yield is significantly increased in benign diseases compared to EBUS-TBNA alone.

\section{Financial Disclosure and Conflicts of Interest}

The authors report that there are no potential conflicts of interest with any companies/organizations whose products or services may be discussed in this article.

\section{References}

-1 Yasufuku K, Pierre A, Darling G, de Perrot M, Waddell T, Johnston M, da Cunha Santos G, Geddie W, Boerner S, Le LW, Keshavjee S: A prospective controlled trial of endobronchial ultrasound-guided transbronchial needle aspiration compared with mediastinoscopy for mediastinal lymph node staging of lung cancer. J Thorac Cardiovasc Surg 2011;142:13931400.

-2 Rintoul RC, Skwarski KM, Murchison JT, Wallace WA, Walker WS, Penman ID: Endobronchial and endoscopic ultrasound-guided real-time fine-needle aspiration for mediastinal staging. Eur Respir J 2005;25:416-421.

- 3 Herth FJ, Eberhardt R, Vilmann P, Krasnik M, Ernst A: Real-time endobronchial ultrasound guided transbronchial needle aspiration for sampling mediastinal lymph nodes. Thorax 2006;61:795-798.

- 4 Herth FJ, Eberhardt R, Krasnik M, Ernst A: Endobronchial ultrasound-guided transbronchial needle aspiration of lymph nodes in the radiologically and positron emission tomography-normal mediastinum in patients with lung cancer. Chest 2008;133:887-891.

5 Lim E, Baldwin D, Beckles M, Duffy J, Entwisle J, Faivre-Finn C, Kerr K, Macfie A, McGuigan J, Padley S, Popat S, Screaton N, Snee M, Waller D, Warburton C, Win T: Guidelines on the radical management of patients with lung cancer. Thorax 2010;65: iii1-iii27.

6 Kennedy MP, Jimenez CA, Bruzzi JF, Mhatre AD, Lei X, Giles FJ, Fanning T, Morice RC, Eapen GA: Endobronchial ultrasound-guided transbronchial needle aspiration in the diagnosis of lymphoma. Thorax 2008;63:360365.

7 Steinfort DP, Conron M, Tsui A, Pasricha SR, Renwick WE, Antippa P, Irving LB: Endobronchial ultrasound-guided transbronchial needle aspiration for the evaluation of suspected lymphoma. J Thorac Oncol 2010;5: 804-809.
> Wong M, Yasufuku K, Nakajima T, Herth FJ, Sekine Y, Shibuya K, Iizasa T, Hiroshima K, Lam WK, Fujisawa T: Endobronchial ultrasound: new insight for the diagnosis of sarcoidosis. Eur Respir J 2007;29:1182-1186.

\$9 Garwood S, Judson MA, Silvestri G, Hoda R, Fraig M, Doelken P: Endobronchial ultrasound for the diagnosis of pulmonary sarcoidosis. Chest 2007;132:1298-1304.

10 Herth FJ, Morgan RK, Eberhardt R, Ernst A: Endobronchial ultrasound-guided miniforceps biopsy in the biopsy of subcarinal masses in patients with low likelihood of non-small cell lung cancer. Ann Thorac Surg 2008;85: 1874-1878.

11 Chrissian A, Misselhorn D, Chen A: Endobronchial-ultrasound guided miniforceps biopsy of mediastinal and hilar lesions. Ann Thorac Surg 2011;92:284-288.

12 Oki M, Saka H, Sako C: Bronchoscopic miniforceps biopsy for mediastinal nodes. J Bronchology Interv Pulmonol 2004;11:150-153.

13 Prakash UB: A better bronchoscopic technique to obtain diagnostic tissue from mediastinal lymph nodes. J Bronchology Interv Pulmonol 2005;12:1-2.

14 Franke KJ, Bruckner C, Szyrach M, Ruhle KH, Nilius G, Theegarten D: The contribution of endobronchial ultrasound-guided forceps biopsy in the diagnostic workup of unexplained mediastinal and hilar lymphadenopathy. Lung 2012;190:227-232.

15 Gasparini S, Zuccatosta L, Sediari M, Mei F: Pilot feasibility study of transbronchial needle forceps - a new tool for obtaining histology samples from mediastinal subcarinal lymph nodes. J Bronchology Interv Pulmonol 2009; 16:183-187.

16 Dasgupta A, Mehta AC, Wang KP: Transbronchial needle aspiration. Semin Respir Crit Care Med 1997;18:571-581.
17 Eisenach KD, Cave MD, Bates JH, Crawford JT: Polymerase chain reaction amplification of a repetitive DNA sequence specific for $M y$ cobacterium tuberculosis. J Infect Dis 1990; 161:977-981.

18 Yasufuku K, Chiyo M, Sekine Y, Chhajed PN, Shibuya K, Iizasa T, Fujisawa T: Real-time endobronchial ultrasound-guided transbronchial needle aspiration of mediastinal and hilar lymph nodes. Chest 2004;126:122128.

19 Holty JE, Kuschner WG, Gould MK: Accuracy of transbronchial needle aspiration for mediastinal staging of non-small cell lung cancer: a meta-analysis. Thorax 2005;60:949955 .

20 Hwangbo B, Lee GK, Lee HS, Lim KY, Lee SH, Kim HY, Lee HS, Kim MS, Lee JM, Nam BH, Zo JI: Transbronchial and transesophageal fine-needle aspiration using an ultrasound bronchoscope in mediastinal staging of potentially operable lung cancer. Chest 2010; 138:795-802.

21 Nakajima T, Kimura H, Takeuchi K, Soda M, Mano H, Yasufuku K, Iizasa T: Treatment of lung cancer with an ALK inhibitor after EML4-ALK fusion gene detection using endobronchial ultrasound-guided transbronchial needle aspiration. J Thorac Oncol 2010; 5:2041-2043.

-22 Nakajima T, Yasufuku K, Suzuki M, Hiroshima K, Kubo R, Mohammed S, Miyagi Y, Matsukuma S, Sekine Y, Fujisawa T: Assessment of epidermal growth factor receptor mutation by endobronchial ultrasound-guided transbronchial needle aspiration. Chest 2007;132: 597-602.

23 Nakajima T, Yasufuku K, Kurosu K, Takiguchi Y, Fujiwara T, Chiyo M, Shibuya K, Hiroshima K, Nakatani Y, Yoshino I: The role of EBUS-TBNA for the diagnosis of sarcoidosis - comparisons with other bronchoscopic diagnostic modalities. Respir Med 2009;103: 1796-1800. 
24 Herth F, Schuler H, Gompelmann D, Kahn N, Gasparini S, Ernst A, Schuhmann M, Eberhardt R: EBUS-guided lymph node biopsy (EBUS-TBNB) with a transbronchial needle forceps (TBNF) - a pilot study. Eur Respir J 2012;39:373-377.

-25 Agarwal R, Srinivasan A, Aggarwal AN, Gupta D: Efficacy and safety of convex probe EBUS-TBNA in sarcoidosis: a systematic review and meta-analysis. Respir Med 2012; 106:883-892.
6 Costabel U, Hunninghake GW: ATS/ERS/ WASOG statement on sarcoidosis. Sarcoidosis Statement Committee. American Thoracic Society. European Respiratory Society. World Association for Sarcoidosis and Other Granulomatous Disorders. Eur Respir J 1999; 14:735-737.

27 Porte H, Roumilhac D, Eraldi L, Cordonnier C, Puech P, Wurtz A: The role of mediastinoscopy in the diagnosis of mediastinal lymphadenopathy. Eur J Cardiothorac Surg 1998;13: 196-199.

28 Cybulsky IJ, Bennett WF: Mediastinoscopy as a routine outpatient procedure. Ann Thorac Surg 1994;58:176-178.
29 Reich JM, Brouns MC, O'Connor EA, Edwards MJ: Mediastinoscopy in patients with presumptive stage I sarcoidosis: a risk/benefit, cost/benefit analysis. Chest 1998;113:147-153.

30 Ernst A, Anantham D, Eberhardt R, Krasnik M, Herth FJ: Diagnosis of mediastinal adenopathy-real-time endobronchial ultrasound guided needle aspiration versus mediastinoscopy. J Thorac Oncol 2008;3:577.

31 Stather DR, MacEachern P, Chee A, Tremblay A: Safety of endobronchial ultrasound-guided transbronchial needle aspiration for patients taking clopidogrel: a report of 12 consecutive cases. Respiration 2012;83:330-334. 